\title{
RELAÇÃO DOS FATORES \\ COMPORTAMENTAIS NOS \\ MÉTODOS DE CONTABILIDADE \\ GERENCIAL NOS HOTÉIS DE \\ SANTA CATARINA
}

RELATIONSHIP OF BEHAVIORAL FACTORS IN ACCOUNTING METHODS OF MANAGEMENT IN SANTA CATARINA HOTEL

RELACIÓN DE LOD FACTORES COMPORTAMIENTO EN LOS MÉTODOS CONTABLES

DE GESTIÓN EN HOTELES EN SANTA CATARINA

\section{Camila Freitas Sant' Ana}

Professora do Curso de Ciências Contábeis da Faculdade de Itapiranga (FAI) Professora do Curso de Ciências Contábeis da Universidade Regional Integrada do Alto Uruguai e das Missões (URI)

Mestre em Ciências Contábeis pelo Programa de Pós-Graduação em Ciências Contábeis (PPGCC) da Universidade Regional de Blumenau (FURB)

mila-internacional@hotmail.com

\section{Cleston Alexandre dos Santos}

Professor do Curso de Ciências Contábeis da Universidade Federal de Mato Grosso do Sul (UFMS), Campus de Três Lagoas (CPTL)

Doutorando em Ciências Contábeis e Administração pelo Programa de PósGraduação em Ciências Contábeis (PPGCC) da Universidade Regional de Blumenau (FURB)

Mestre em Contabilidade pelo Programa de Pós-Graduação em Ciências Contábeis (PPGCONT) da Universidade Federal do Paraná (UFPR) cleston.alexandre@hotmail.com 


\title{
Paulo Roberto da Cunha
}

\author{
Professor e Coordenador do Programa de Pós-Graduação em Ciências Contábeis
} (PPGCC) da Universidade Regional de Blumenau (FURB)

Professor do Curso de Ciências Contábeis da Universidade do Estado de Santa Catarina (UDESC)

Doutor em Ciências Contábeis e Administração pelo PPGCC da FURB Mestre em Ciências Contábeis pelo PPGCC da FURB pauloccsa@furb.br

\author{
Data de Submissão: 16/05/2016 \\ Data de Aprovação: 21/10/2016
}

\begin{abstract}
RESUMO: Fatores comportamentais podem evidenciar como os gestores absorvem informações e como utilizam os métodos de contabilidade gerencial na condução de suas decisões organizacionais na atividade hoteleira. Assim, o objetivo deste trabalho é verificar a relação entre os fatores comportamentais e a utilização dos métodos de contabilidade gerencial nos hotéis de Santa Catarina. O método de pesquisa utilizado caracteriza-se como descritivo, quantitativo, realizado por meio de pesquisa survey. A amostra foi composta por 32 gerentes de hotéis de Santa Catarina, selecionados com base na população de 149 hotéis vinculados a Associação Brasileira da Indústria de Hotéis do Estado de Santa Catarina (ABIH-SC). Os dados foram analisados por meio de estatísticas descritivas e correlação canônica. Os resultados evidenciaram que os respondentes dos hotéis investigados apresentam-se principalmente com fatores comportamentais voltados a uma visão com maior grau explorador em relação ao nível de Abertura, maior grau de flexibilidade em relação a Consciência, maior grau de Extroversão em se tratando da Extroversão, maior grau de parceria no que tange a Sociabilidade e Agradabilidade e maior grau de reatividade em relação ao Neuroticismo. Por fim, foi constatado que os fatores comportamentais identificados influenciam na utilização dos métodos de contabilidade gerencial nas organizações hoteleiras de Santa Catarina, com evidenciação de alta correlação entre as variáveis analisadas.
\end{abstract}

PALAVRAS-CHAVE: Fatores comportamentais. Métodos gerenciais. Gestão de hotéis.

ABSTRACT: Behavioral factors may show how managers absorb information and how they use management accounting methods in the conducting its organizational decisions in the hotel business. The objective of this study is to investigate the relationship between behavioral factors and the use of management accounting methods in hotels of Santa Catarina. The research method used is characterized as descriptive, quantitative, conducted through survey research. The sample consisted of 32 managers of hotels in Santa Catarina, selected based on the population of 149 hotels linked to the Brazilian Association of the Industry of the State of Santa Catarina Hotels (ABIH-SC). Data were analyzed using descriptive statistics and canonical correlation. The results showed that the respondents of the investigated hotels are presented mainly with behavioral factors aimed at a vision the greatest explorer degree on the level of opening, the greater degree of flexibility in relation to consciousness, greater degree of extroversion in the case of extroversion, greater degree of partnership in relation to sociability and Agreeableness and greater degree of reactivity with 
respect to Neuroticism. Finally, it was found that the identified behavioral factors influence the use of management accounting methods in hotel organizations of Santa Catarina, with high correlation disclosure between the variables.

KEYWORDS: Behavioral Factors. Management Methods. Hotel Management.

RESUMEN: Factores de comportamiento pueden mostrar cómo los gerentes de absorber la información y cómo utilizar los métodos de contabilidad de gestión en la realización de sus decisiones de organización en el negocio hotelero. El objetivo de este estudio es investigar la relación entre los factores de comportamiento y el uso de métodos de contabilidad de gestión en hoteles de Santa Catarina. El método de investigación utilizado se caracteriza por ser descriptiva, cuantitativa, realizada a través de la investigación por encuestas. La muestra estuvo constituida por 32 gerentes de hoteles en Santa Catarina, seleccionados en base a la población de 149 hoteles vinculados a la Asociación Brasileña de la Industria del Estado de Santa Catarina Hoteles (ABIH-SC). Los datos fueron analizados utilizando estadística descriptiva y correlación canónica. Los resultados mostraron que los encuestados de los hoteles investigados se presentan principalmente con factores de comportamiento dirigidas a una visión grado explorador más grande en el nivel de apertura, el mayor grado de flexibilidad en relación con la conciencia, un mayor grado de extraversión en el caso de la extroversión, mayor grado de asociación en relación con la sociabilidad y la agradabilidad y un mayor grado de reactividad con respecto a neuroticismo. Por último, se encontró que los factores de comportamiento identificados influyen en el uso de los métodos de contabilidad de gestión en las organizaciones de hoteles de Santa Catarina, con la divulgación alta correlación entre las variables.

PALABRAS CLAVE: Factores de comportamiento. Métodos de gestión. La dirección del hotel.

\section{INTRODUÇÃO}

A s organizações têm sobrevivido aos períodos de grandes mudanças no mercado, em função de fatores como a globalização e o avanço tecnológico. O processo de gestão das organizações, inclusive nas atividades de hotelaria, tem apresentado diversas alterações e adaptações ao longo dos anos, principalmente no âmbito da forma de atuação, o que faz as organizações adotarem métodos de gestão que melhor se adéque as necessidades informacionais (Adler, Everett, \& Waldron, 2000). Autores como Mondo e Costa (2013), Beuren e Fiorentin (2014) e Marquesan, Guzzo, Zawislak, \& Tello-Gamarra, (2015), argumentam que a globalização, avanços tecnológicos, alterações no contexto social e no mercado competitivo, tem gerado incertezas no processo de decisão das empresas, o que pode refletir na sua permanência no mercado junto aos concorrentes.

Mosimann e Fisch (1999) evidenciam que o processo de gestão das organizações contempla um conjunto de processos decisórios, que são 
ISSN: 1983-7151

essenciais para administração de um negócio. Os autores destacam que o processo de gestão compreende o planejamento, execução e controle, cujo processo tem como propósito possibilitar as organizações alcançarem seus resultados de forma coordenada e orientados pela filosofia e pelo modelo de gestão institucional, o que em muitas situações, exigem mudanças internas.

Para acompanhar o processo de mudanças, torna-se necessário que o controller e o gestor de organizações hoteleiras tenham condições de se adaptar a tais mudanças, o que exige a busca por novas tecnologias e métodos de contabilidade gerencial contemporâneos (Adler, Everett, \& Waldron, 2000). Oliveira, Pereira, \& Ribeiro (2008) argumentam que a contabilidade gerencial é considerada relevante na tomada de decisão das unidades hoteleiras, o que justifica o crescente interesse pelos métodos gerenciais na gestão dos estabelecimentos hoteleiros.

Para Pavlatos e Paggios (2009), como a atividade hoteleira tem atuado em um mercado cada vez mais competitivo, essa concorrência tem influenciado o interesse e a adoção de modernas técnicas de gestão. De acordo com Mia e Patiar (2001), na atividade hoteleira os gestores têm demandado por um maior número de informações, e em muitas situações referem-se a informações diferentes de outras atividades econômicas, pois estão suscetíveis às grandes oscilações da procura, possuem elevada estrutura de custos fixos, trabalham com produtos perecíveis, com produtos e serviços diversificados em função da heterogeneidade dos clientes, e ainda, atuam em mercados altamente competitivos.

A demanda por informações e por métodos de contabilidade gerencial, como a sua utilização no processo de gestão, pode variar de pessoa para pessoa, em função de fatores comportamentais cognitivos (Gosling, Rentfrow, \& Swann, 2003). Tendo em vista que de acordo com Bandura (1977), a teoria social cognitiva auxilia na explicação quanto ao funcionamento humano, derivado da interação bidirecional entre os fatores cognitivos e pessoais, fatores ambientais e fatores comportamentais no desenvolvimento e nas ações humanas.

Frente ao exposto, Gosling etal. (2003) consideramosfatorescomportamentais cognitivos do profissional para avaliar o papel que conduzirá nas atividades gerenciais, ou seja, evidenciam a maneira em que os gestores e controllers 
administram e processam as informações em torno da solução de problemas e das decisões tomadas.

Diante disso, Emsley e Chung (2010) evidenciam que fatores comportamentais influenciam em mudanças, o que pode revelar que gestores e controllers com características de inovação, pró-ativos e atuantes são os que possuem maior probabilidade de apresentar esforços para desenvolver mudanças efetivas no processo organizacional.

Neste contexto, propõe-se como questão de pesquisa: qual a relação dos fatores comportamentais na utilização dos métodos de contabilidade gerencial nos hotéis de Santa Catarina? Decorrente da questão de pesquisa surge o objetivo de verificar a relação dos fatores comportamentais na utilização dos métodos de contabilidade gerencial nos hotéis de Santa Catarina.

O campo acadêmico apresenta estudos que buscam analisar fatores comportamentais e práticas de gestão (Gosling, Renfrow, \& Swann, 2003; Rouwelaar \& Bots, 2008; Emsley \& Chung, 2010; Faria, Trigueiros, \& Ferreira, 2012; Teles, Lunkes, \& Espejo, 2012), e abre espaço para novas pesquisas, com novas evidenciações, relações e percepções. Bem como, ainda Faria et al. (2012) destacam a necessidade de estudos que identifiquem os fatores que influenciam a contabilidade de gestão nas unidades hoteleiras.

Estudos em contabilidade gerencial têm revelado atenção às organizações do setor industrial, o que tem demonstrado pouca abrangência do estudo do setor de serviços, principalmente o hoteleiro (Faria et al., 2012; Pellinen, 2003). Sharma (2002) chama a atenção para a necessidade e oportunidade de novos estudos para se identificar fatores que apontam a adoção e uso dos sistemas de controle de gestão nos hotéis, em face da representatividade econômica.

A literatura tem mostrado que o turismo é considerado como um dos principais responsáveis pela movimentação da economia mundial, e como representante em destaque do setor de serviços, os empreendimentos de hospedagem, como hotéis, tem uma função primordial no desenvolvimento deste segmento (Mondo \& Costa, 2013; Marquesan et al., 2015). Nesse contexto, o Estado de Santa Catarina é tratado como sendo um dos principais receptores de turistas no Brasil, tendo reconhecimento em função das belezas 
naturais e pelas acomodações disponíveis, cuja demanda tem contribuído para expansão das redes hoteleiras e crescimento econômico dos municípios do Estado (Mondo \& Costa, 2013).

Como o setor hoteleiro em particular tem sido evidenciado como uma das atividades em destaque pelo seu crescimento econômico nos últimos anos (Marquesan et al., 2015), torna-se um importante campo de pesquisa no Brasil. Autores como Wilke e Rodrigues (2013) e Zonatto, Schuh e Zonatto (2014) argumentam que o segmento hoteleiro tem seu destaque pelo alto grau de competição que há entre os concorrentes desse setor, o que impõe a necessidade dos hotéis terem gestores com condições de desenvolver ações que contribuam para uma melhor tomada de decisão, voltadas aos crescimento de suas empresas. Dessa forma, tais condições são propícias para a condução desta investigação.

A estrutura do trabalho contempla a fundamentação teórica, em seguida, a descrição dos procedimentos metodológicos, a apresentação, análise e discussão dos dados da pesquisa, e por fim, as considerações finais.

\section{REFERENCIAL TEÓRICO}

Na revisão de literatura são abordados os métodos de contabilidade gerencial utilizados na gestão dos hotéis, e por último os fatores comportamentais segundo o Ten Itens Personality Inventory [TIPI].

\section{GESTÃO E OS MÉTODOS DE CONTABILIDADE GERENCIAL NA} ATIVIDADE HOTELEIRA

A gestão de empresas hoteleiras é uma atividade que exige atenção dos controllers, dado a complexidade dos processos relacionados a prestação de serviços (Sharma, 2002). Neste sentido, Fitzsimmons e Fitzsimmons (2014) destacam que as empresas de prestação de serviços devem identificar as particularidades exclusivas de seu setor de atuação para assim prover-se de ferramentas hábeis para um bom gerenciamento do negócio, pois a falta de 
informações gerenciais pode prejudicar o sucesso ou mesmo proporcionar o fracasso da organização.

Para Horngren, Foster e Datar (2004) o processo de gestão de hotéis compreende a diversas atividades que variam desde o planejamento ao controle, por exemplo as decisões de planejamento e controle das rotinas diárias do hotel. Entretanto, Petrocchi (2007) salienta que o desafio da gestão do hotel não deve ser preocupar-se somente com os aspectos internos, mais também com os aspectos externos ligados a organização, que possam vir a influenciar nas escolhas de seus clientes e consequentemente afetar seu desempenho.

A contabilidade gerencial auxilia como um forte instrumento de gestão, disponibilizando diversas técnicas, métodos e procedimentos para a obtenção de informações relevantes para dar suporte ao processo de tomada de decisão, quanto ao planejamento, a avaliação, ao controle de recursos e das atividades operacionais, voltados ao atendimento dos objetivos estabelecidos pela gestão da organização (Iudícibus, 1998; Jiambalvo, 2002; Martin, 2002; Guerreiro, Frezatti, Lopes, \& Pereira, 2005; Frezatti, Aguiar, \& Guerreiro, 2007; Cabrelli \& Ferreira, 2007).

O estabelecimento de controles gerenciais nas organizações hoteleiras é um elemento fundamental para promover o desempenho das mesmas (Lunkes, 2009), pois estes sistemas representam um ganho informacional essencial para uma gestão eficiente e eficaz, em virtude de que por meio destes sistemas informacionais a gestão hoteleira seja capaz de construir conhecimentos e habilidades para estabelecer as práticas de gerenciamento dos dados e das informações úteis, não somente para os gerentes de departamento mais como também para os principais executivos da organização (Rutherford, 2004).

Conforme as características de cada hotel, principalmente do tipo de serviço prestado, como venda de estadas nos apartamentos, serviços de lavanderia, etc., destaca-se que os gestores dos hotéis, gerentes gerais ou gerentes de departamento, deparam-se com o objetivo de administrar uma gama de processos (Petrocchi, 2007), gerando uma necessidade de prover-se de métodos gerenciais para desempenharem sua função. 
Assim, conforme ressaltado por Ferreira e Otley (2006), os métodos e artefatos de contabilidade gerencial referem-se a um conjunto de Sistemas de Controle Gerencial elencados na dimensão de desenho da organização, sob o qual após a entrada de dados são capazes de gerar informações para o processo de tomada de decisão.

De acordo com Lunkes (2009), os gestores devem estar atentos aos diversos tipos de controles que podem ser elencados no estabelecimento de um sistema de controle adequado e capaz de salvaguardar as operações e os ativos da organização, que para Almeida (1996) deve estabelecer-se com base em dados confiáveis e relevantes para a gestão do negócio, do qual Lunkes (2009) enfatiza como fundamental a observação dos controles organizacionais e estruturais, sistemas de informação e controle de métodos e procedimentos para o alcance de uma gestão eficaz.

De acordo com Araújo (2001, p.27), o gestor em hotelaria é o responsável pela definição e a avaliação dos produtos e serviços que podem proporcionar vantagens competitivas. Sendo que a principal meta da gestão dos hotéis em uma perspectiva financeira, refere-se a obtenção de lucro e a capacidade de gerar retorno do capital para os investidores (Rosa \& Lunkes, 2006).

Para alcançar os objetivos organizacionais pré-determinados, é necessário que o gestor, por meio do sistema de contabilidade gerencial em que fará a escolha dos métodos de contabilidade gerencial, leve em consideração as diferenças das necessidades informacionais dos stakeholders (Faria et. al., 2012). Por métodos de contabilidade gerencial, entende-se como sendo sistemas de informação, modelos de gestão, sistemas de custeio, filosofias implantadas pelas organizações e conceitos de mensuração e avaliação de desempenho, que fazem parte do processo de gestão das atividades organizacionais (Frezatti, 2005; Guerreiro, Cornachione Jr," \& Soutes, 2011).

Os métodos considerados tradicionais focam na determinação do custo e controle financeiro, empregado pelo uso da tecnologia de orçamento e contabilidade de custos, como também centrado na disponibilização de informação para o processo de planejamento e controle gerencial, em função do uso de técnicas de análise de decisões e contabilidade por responsabilidade. 
Os métodos modernos são voltados para redução de perdas de recursos nos processos de fabricação e/ou prestação de serviços, com o propósito de análise de processos e a gestão de custos e com ênfase na geração e/ou a criação de valor pelo uso contínuo e de forma efetiva dos recursos disponíveis, visando agregar valor para o cliente e a inserção de inovação organizacional (Soutes, 2006; Faria et al., 2012; Xavier \& Carmo Filho, 2015).

Assim, como práticas de contabilidade gerencial, os de métodos tradicionais utilizados na gestão organizacional compreendem o Custeio Padrão, Custeio por Absorção, Custeio Variável, Descentralização, Moeda Constante, Orçamento, Preço de Transferência, Retorno sobre Investimento e Valor Presente. Já como métodos de contabilidade gerencial modernos tem-se o Balanced Scorecard $[B S C]$, Benchmarking, Custeio Baseado em Atividades [ABC], Custeio Meta, Gestão Baseada em Atividade [ABM], Economic Value Added [EVA], Gestão Baseada em Valor [VBM], Just in Time [JIT], Kaizen, Planejamento Estratégico, Simulação e Teoria das Restrições (Cunha \& Frezatti, 2002; Rosa \& Lunkes, 2006; Vieira \& Souza, 2005; Castro, Santos, Souza, \& Lunkes, 2012; Faria et al., 2012, Codesso, Lunkes, \& Suave, 2013; Xavier \& Carmo Filho, 2015).

No estudo de Faria et al. (2012) os resultados revelam uma elevada adoção de métodos tradicionais de contabilidade gerencial, como é o caso do orçamento, mas uma reduzida utilização do custeio baseado em atividades, da análise de rentabilidade, do Balanced Scorecard [BSC] e de outras técnicas contemporâneas. Desta forma, ressalta-se que esses métodos são administrados pelo gestor do hotel, sendo que neste aspecto Borinelli (2006) destaca o relacionamento com a área de psicologia, pois os indivíduos tomadores de decisão por meio de seus atos comportamentais podem acabar influenciando no controle e na gestão organizacional.

Neste sentido, percebe-se que fatores comportamentais como os fatores pessoais cognitivos podem promover impactos sobre a forma como os gestores utilizam-se dos métodos gerenciais na gestão dos hotéis, em virtude de que Emsley e Chung (2010) salientam que os estilos cognitivos dos gestores refletem no modo como os mesmos organizam e processam as informações para o processo de tomada de decisão. 
Qualquer que seja a atividade desenvolvida, o conhecimento empírico e a experiência, é entendida como um possível diferencial para o processo de gestão organizacional. Na atividade hoteleira não é diferente, o gestor da organização pode conter elementos que gerem influencia na sua maneira de agir e decidir (Teles et al., 2012). Nesse contexto, diante das variáveis internas e externas que necessitam de um melhor controle do gestor, exigem que esse profissional detenha habilidades e comportamentos adequados para o desenvolvimento das atividades organizacionais pré-estabelecidas (Nascimento \& Reginato, 2010).

Cada gestor ou grupo de pessoas, é detentor de um padrão de comportamento, que contempla pensamento, sentimentos, atitudes, como também outros atributos (Hall, Lindzey, \& Campbell, 2000; Carvalho, Nunes, \& Prime, 2012). Esse padrão de comportamento é compreendido como uma forma de funcionamento de cada ser humano, que consiste ao seu padrão de personalidade, ou seja, é entendido como sendo a combinação de diferentes sistemas relacionados aos atributos psicológicos (Mayer, 2005).

Fatores comportamentais refletem a forma com que os gestores organizam e transmitem as informações para decisões gerenciais e na solução dos problemas inerentes a atividade, sendo que diferente dos profissionais tradicionais, os com tendência inovadora e proativas, possuem maiores chances de gerar esforços para mudanças na contabilidade gerencial (Emsley \& Chung, 2010).

Para entender o padrão de comportamento das pessoas, o modelo dos Cinco Grandes Fatores [CGF] tem sido um dos mais adotados e citados nos estudos (Digman, 2002; Carvalho et al., 2012). O modelo CGF é considerado simples e robusto para descrever as características de personalidade, como também é possível de ser adaptado e aplicado em diversas culturas e em outros campos além da psicologia (Lucas, Diener, Grob, Suh, \& Shao, 2000).

Com base no modelo dos Cinco Grandes Fatores [CGF], também conhecido como 'big-five' - cinco fatores de personalidade de Howard e Howard (1995), 
Gosling, Renfrow e Swann (2003) desenvolveram um instrumento para medir o comportamento, com dez características de personalidade, cujo modelo considerado de fácil aplicação e robusto, recebeu o nome de TIPI [Ten Itens Personality Inventory]. Segundo Robins, Tracy, Trzesniewski, Potter, \& Gosling (2001), os instrumentos de pesquisa mais simples e breves possibilitam a eliminação de itens redundantes do instrumento de teste, como também influenciam na redução da fadiga participantes do teste, e dessa forma, defendem o desenvolvimento e a aplicação de instrumentos mais objetivos.

O modelo TIPI, que pressupõe que as pessoas são diferentes nas personalidades, apresenta uma classificação de cinco grupos de personalidades com duas características para cada grupo, totalizando 10 características, conforme Tabela 1 (Gosling et al., 2003).

Tabela 1 - Grupos e características comportamentais

\begin{tabular}{c|c|l|l}
\hline Grupos & \multicolumn{3}{|c}{ Características } \\
\hline Abertura $(\mathrm{A})$ & $\begin{array}{c}\text { o grau em que é curioso (explorador) } \\
(\mathrm{A}+)\end{array}$ & Versus & o grau em que é conservador (A-) \\
\hline Consciência (C) & o grau em que é disciplinado (C+) & Versus & o grau em que é flexível (C-) \\
\hline Extroversão (E) & o grau em que é extrovertido (E+) & Versus & o grau em que é introvertido (E-) \\
\hline $\begin{array}{c}\text { Sociabilidade ou } \\
\text { Agradabilidade (S) }\end{array}$ & o grau em que é parceiro $(\mathrm{S}+)$ & Versus & grau em que é competidor (S-) \\
\hline Neuroticismo (N) & o grau em que é reativo $(\mathrm{N}+)$. & Versus & o grau em que é resiliente (N-) \\
\hline
\end{tabular}

Fonte: Gosling et al. (2003).

Conforme a Tabela 1, o instrumento TIPI mensura os cinco grupos de personalidade com duas características para cada grupo, sendo uma positiva (+) e uma negativa (-). De acordo com Gosling et al. (2003):

O grupo Abertura (A) consiste na possibilidade de reconhecimento de novas experiências ou formas de fazer algo, sendo que os indivíduos que possuem alta pontuação nessa dimensão são denominados de exploradores, pois são voltados a inovação, criatividade e contrários a atividades consideradas simples e mecânicas. Na constatação de uma pontuação baixa, revela características conservadoras e com interesses mais restritos;

O grupo Consciência $(C)$ revela o grau de dedicação que um indivíduo tem para alcançar os objetivos, ou seja, o grau de organização, persistência, controle 
e motivação. Pessoas com alta pontuação nessa dimensão são disciplinadas e focadas nas tarefas em execução. Já as com baixa pontuação tendem a aproximar-se dos objetivos em ambientes que sejam mais descontraídos, são consideradas como pessoas flexíveis e que são de fácil adaptabilidade as mudanças de circunstancias;

Extroversão (E) compreende a quantidade e intensidade das interações interpessoais preferidas, sendo que pessoas com alta pontuação tem preferência por estar perto de outras, e ainda, é normal participarem de diversas atividades. As com baixa pontuação tem preferência por trabalharem sozinhas, mas são calmas, sérias e reservadas;

Sociabilidade ou Agradabilidade (S) consiste em uma dimensão interpessoal que se refere a um conjunto de habilidades sociais e ao grau de submissão aos outros. Nesse contexto, pessoal com alta pontuação tendem a cooperar, são agradáveis, receptivas e tolerantes. Já as com baixa pontuação, tendem a serem desconfiadas, hostis e não gostar de desenvolver trabalhos em grupo;

Por fim, Neuroticismo (N) refere-se a estabilidade emocional, compreende o grau de resposta a situações estressantes. Pessoal com elevada pontuação tendem a ser mais inseguras, preocupadas, atentas, emotivas, e portanto, sentem mais estresse e são reativas. Já as com baixa pontuação são mais calmas e seguras, não praticam ações em função de sentimentos e emoções, mas sim em função de fatos concretos e reais.

Com base nas características comportamentais apresentadas por Gosling, et al. (2003) no modelo TIPI, Rouwelaar e Bots (2008) apontam que os grupos abertura, sociabilidade e extroversão apresentam uma associação positiva com o grau de envolvimento do controller na gestão, porém nos grupos consciência e neuroticismo a associação é negativa. Portanto, a atuação do profissional de contabilidade apresenta uma relação com o crescimento organizacional, de tal forma que a postura assumida seja gerencial e proativa, e vai além das práticas rotineiras da escrituração contábil e da prestação de informações.

Há muitos anos o fator comportamental já fazia parte de discussão sobre possíveis relações no processo de gestão: Wixon, Kell e Bedford (1943) com a argumentação de que situações técnicas de mensuração contábil são 
influenciadas por características comportamentais, como capacidade de autocontrole, de comunicação e negociação; Bower (1957) com a discussão da necessidade do profissional de contabilidade em conciliar conhecimentos técnicos específicos com fatores comportamentais, tais como capacidade de comunicação e de relacionamento; Hardern (1995) com a ênfase de que estudos de contabilidade devem ter uma direção para competências, fatores explicativos das mudanças organizacionais, das práticas gerenciais, o que deve demandar maior foco em abordagens comportamentais.

No estudo de Teles et al. (2012) foi identificado o nível de percepção dos controllers da região sul do Brasil acerca de suas habilidades, nas perspectivas pessoal, contingencial e comportamental. Os resultados apontaram que as habilidades pessoais com maiores médias estão centradas na habilidade na tomada de decisão, competência de solucionar problemas e liderança. Foi evidenciado também que as habilidades de comunicação dos pesquisados prevaleceram sobre as habilidades técnicas e rotinas de trabalho, cujo resultado também demonstra um destaque para as atividades desenvolvidas em grupo.

\section{PROCEDIMENTOS METODOLÓGICOS}

Com o intuito de verificar a relação dos fatores comportamentais no processo de gestão de hotéis de Santa Catarina, quanto ao objetivo realizouse uma pesquisa descritiva, quanto aos procedimentos uma pesquisa survey e em relação à abordagem do problema como quantitativa.

A população do presente estudo consiste em 149 hotéis do Estado de Santa Catarina, vinculados à Associação Brasileira da Indústria de Hotéis do Estado de Santa Catarina, conforme informações no site da instituição (ABIH-SC, 2015). A amostra foi composta por 32 hotéis que voluntariamente responderam à pesquisa, assim, trata-se de uma amostra não aleatória, gerada por acessibilidade. A amostra caracteriza-se como não probabilística, pelo fato de os questionários terem sido enviados a todos os hotéis que compõem a população efetiva, e de não ter como definir de forma prévia quais participarão (Fonseca \& Martins, 1996). 
Na Tabela 2, são apresentadas as variáveis que representam os fatores comportamentais do gestor e os métodos gerenciais que foram evidenciados, de acordo com a revisão de literatura realizada sobre a temática.

Tabela 2 - Constructo da pesquisa

\begin{tabular}{|c|c|c|c|}
\hline Variáveis & Sub-variáveis & Questões nº & Autores \\
\hline \multirow{5}{*}{$\begin{array}{c}\text { Fatores } \\
\text { comportamentais } \\
\text { (TIPI) }\end{array}$} & Abertura & Q13 \& Q14 & \multirow{5}{*}{ Gosling et al. (2003) } \\
\hline & Consciência (consolidação) & Q15 \& Q16 & \\
\hline & Extroversão & Q17 \& Q18 & \\
\hline & Sociabilidade & Q19 \& Q20 & \\
\hline & Neuroticismo (estabilidade emocional) & Q21 \& Q22 & \\
\hline \multirow{9}{*}{$\begin{array}{l}\text { Métodos } \\
\text { Gerenciais } \\
\text { Tradicionais }\end{array}$} & Custeio Padrão & Q23 & \multirow{21}{*}{$\begin{array}{l}\text { Cunha \& Frezatti, (2002); } \\
\text { Rosa \& Lunkes, (2006); } \\
\text { Vieira \& Souza, (2005); } \\
\text { Castro et al., (2012); Faria } \\
\text { et al., (2012), Codesso } \\
\text { et al., (2013); Xavier \& } \\
\text { Carmo Filho, (2015) }\end{array}$} \\
\hline & Custeio por Absorção & Q24 & \\
\hline & Custeio Variável & Q25 & \\
\hline & Descentralização & Q26 & \\
\hline & Moeda Constante & Q27 & \\
\hline & Orçamento & Q28 & \\
\hline & Preço de Transferência & Q29 & \\
\hline & Retorno sobre Investimento & Q30 & \\
\hline & Valor Presente & Q31 & \\
\hline \multirow{12}{*}{$\begin{array}{l}\text { Métodos } \\
\text { Gerenciais } \\
\text { Modernos }\end{array}$} & Balanced scorecard(BSC) & Q32 & \\
\hline & Benchmarking & Q33 & \\
\hline & Custeio Baseado em Atividades (ABC) & Q34 & \\
\hline & Custeio Meta & Q35 & \\
\hline & Gestão Baseada em Atividade (ABM) & Q36 & \\
\hline & EVA (Economic Valueadded) & Q37 & \\
\hline & Gestão Baseada em Valor (VBM) & Q38 & \\
\hline & JIT (Just in time) & Q39 & \\
\hline & Kaizen & Q40 & \\
\hline & Planejamento Estratégico & Q41 & \\
\hline & Simulação (de resultados) & Q42 & \\
\hline & Teoria das Restrições & Q43 & \\
\hline
\end{tabular}

Fonte: Elaborado pelo Autor.

A coleta de dados foi realizada por meio de um questionário dividido em quatro seções. A primeira seção compreendeu a caracterização dos respondentes (da questão 1 a 7). A segunda seção compreendeu a caracterização dos hotéis (da questão 8 a 12). A terceira seção envolve a identificação dos fatores comportamentais (da questão 13 a 22). E a última seção identificou o grau de 
adoção dos métodos de contabilidade gerencial no processo de gestão (da questão 23 a 43). Optou-se por encaminhar o questionário via e-mail com contato telefônico prévio junto aos membros que ocupavam os seguintes cargos das entidades objeto de investigação: Administrador, Contador, Controller, Gerente, e outros, desde que fossem gerentes. Os dados foram coletados no período de 02/11/2015 à 14/12/2015.

Destaca-se que o questionário de pesquisa foi estabelecido com base no estudo de Gosling et al. (2003) e de Teles et al. (2012), sob o qual o mesmo foi adaptado para a atender ao objetivo da presente pesquisa. Assim, ressalta-se que o questionário encaminhado por e-mail aos respondentes da pesquisa foi composto por 43 questões fechadas.

Para análise dos dados foram utilizadas a análise das estatísticas descritivas, observando frequências das respostas e a aplicação da correlação canônica para verificar a correlação entre as variáveis da pesquisa, por meio do software STATGRAPHICS Centurion.

\section{ANÁLISE E DISCUSSÃO DOS RESULTADOS}

Neste tópico apresentam-se os resultados da pesquisa realizada.

\section{CARACTERIZAÇÃO DOS RESPONDENTES}

O perfil dos respondentes da amostra apresenta alguns aspectos importantes para a análise de dados. Assim, a Tabela 3 apresenta as principais características evidenciadas na amostra pesquisada, contemplando o gênero e o cargo ou função desempenhada atualmente pelos responsáveis pela gestão dos hotéis de Santa Catarina participantes da pesquisa.

Tabela 3 - Perfil dos respondentes

\begin{tabular}{c|c|ccc}
\hline Gênero & Frequência & $\%$ & Total Freq. & Total \% \\
\hline Masculino & 23 & 72 & \multirow{2}{*}{32} & $100 \%$ \\
\hline Feminino & 9 & 28 & & \\
\hline
\end{tabular}




\begin{tabular}{c|c|c|c}
\hline Cargo/função & Frequência & $\%$ & \\
\hline Administrador & 9 & 28 & \\
\hline Contador & 5 & 16 \\
\hline Controller & 7 & 22 & \multirow{2}{*}{32} \\
\hline Gerente & 10 & 31 & \\
\hline Outros & 1 & 3 \\
\hline
\end{tabular}

Fonte: Dados da pesquisa.

Com base na Tabela 3, observa-se que prevalece na gestão dos hotéis pesquisados indivíduos do gênero masculino com 72\%, com idade entre 28 e 64 anos, sendo a mediana da idade correspondente a 42 anos, sob o qual estão exercendo principalmente a função de gerente do hotel com 39\% dos respondentes, seguidos respectivamente pelos administradores com $28 \%$.

A gestão do hotel, como ressaltado na revisão de literatura realizada, exige dos gestores conhecimentos e habilidades para o estabelecimento de controles gerenciais, que caracterizam-se como elementos fundamentais para promover o desempenho das organizações hoteleiras (Lunkes, 2009).

Assim, os participantes da pesquisa foram questionados em relação ao nível de escolaridade e área de conhecimento, do qual evidenciou-se que $16 \%$ dos respondentes apresenta-se com curso técnico, 28\% com graduação, 50\% com especialização e $6 \%$ com mestrado. Sendo que a área de formação está concentrada com 41\% em Administração, 34\% em Ciências Contábeis, 9\% em Economia e 3\% em Turismo e Hotelaria e 13\% não possui graduação, resultado que colabora para a evidenciação do grau de utilização dos métodos gerenciais no processo de gestão dos hotéis.

Entretanto, ainda foram observadas na amostra pesquisada aspectos relacionados ao tempo de trabalho do gestor no hotel, na função e a idade da organização hoteleira, sob o qual os dados estão apresentados na Tabela 4.

Tabela 4 - Número de respostas por tempo de atuação no hotel, função e idade do hotel

\begin{tabular}{c|c|c|c|c|cc}
\hline \multirow{2}{*}{ Item } & \multicolumn{2}{c}{$\begin{array}{c}\text { Tempo de atuação no } \\
\text { hotel }\end{array}$} & \multicolumn{2}{c}{$\begin{array}{c}\text { Tempo de atuação na atual } \\
\text { função }\end{array}$} & \multicolumn{2}{c}{ Idade do Hotel } \\
\cline { 2 - 7 } & Frequência & $\%$ & Frequência & $\%$ & Frequência & $\%$ \\
\hline Até 5 anos & 5 & 16 & 11 & 34 & 0 & 0 \\
\hline De 6 a 10 anos & 8 & 25 & 14 & 44 & 4 & 13 \\
\hline De 11 a 15 anos & 11 & 34 & 4 & 13 & 6 & 19 \\
\hline De 16 a 20 anos & 4 & 13 & 1 & 3 & 4 & 13 \\
\hline
\end{tabular}


Dor: 10.14210/rtva.v18n2.p154-184

\begin{tabular}{c|c|c|cccc}
\hline Acima de 21 anos & 3 & 9 & 1 & 3 & 19 & 59 \\
\hline Total & $\mathbf{3 2}$ & $\mathbf{1 0 0}$ & $\mathbf{3 2}$ & $\mathbf{1 0 0}$ & $\mathbf{3 2}$ & $\mathbf{1 0 0}$ \\
\hline
\end{tabular}

Fonte: Dados da pesquisa.

De acordo com a Tabela 4, denota-se que os gestores concentram tempo de atuação no hotel entre 11 a 15 anos e tempo na atual função desempenhada aproximadamente de 5 a 10 anos, dados que sinalizam uma certa experiência adquirida dos participantes da pesquisa no quesito de gerenciamento em hotéis, ou seja, conhecimento no desempenho de sua função.

A maioria dos participantes da pesquisa (59\%) representam hotéis com mais de 21 anos de prestação de serviços de hotelaria, com o número de colaboradoresefetivos dos hotéis entre 11 a 280 colaboradores, compreendendo a mediana em 30 colaboradores, mostrando a representatividade e tamanho dos hotéis pesquisados.

De modo que 6 participantes da pesquisa enfatizaram que a taxa média de ocupação da capacidade do hotel em que gerencia é de até $30 \%$. Ainda, 9 gestores salientaram trabalhar com a taxa média de ocupação entre $31 \%$ a $50 \%$ da capacidade do hotel. Outros 6 respondentes indicaram que o hotel para o qual presta seus serviços atua com a taxa de ocupação entre $51 \%$ e $70 \%$ de sua capacidade de hospedagem. Enquanto, 12 respondentes frisaram trabalhar com a taxa média de ocupação do hotel acima de $70 \%$ da capacidade de hospedagens.

Respectivamente a partir da amostra investigada, 4 respondentes indicaram que o hotel para o qual presta seu serviço faz parte de uma rede hoteleira, de modo que os demais 28 respondentes indicaram que os hotéis não fazem parte de redes hoteleiras.

Metade dos respondentes da pesquisa (16) informaram que o hotel não possui um setor interno de contabilidade, resultado que mostra que a contabilidade está sendo realizada por contadores externos a organização, restando assim 16 hotéis que contém a disposição um setor interno responsável pela contabilidade do hotel, fator que pode contribuir para o desenvolvimento e aplicabilidade de mais métodos gerenciais. 


\section{ESTATÍSTICAS DESCRITIVAS E CORRELAÇÃO ENTRE AS VARIÁVEIS}

Dado alguns aspectos importantes para a caracterização dos respondentes, nesta seção abarca-se sobre o grau de utilização dos métodos gerenciais tradicionais e modernos, seguidos dos fatores comportamentais que podem determinar as decisões dos indivíduos responsáveis pela gestão dos hotéis. Assim, a Tabela 5 apresenta o grau de utilização dos métodos gerenciais aplicados a gestão das organizações.

Tabela 5 - Grau de adoção de métodos de contabilidade gerencial no processo de gestão.

\begin{tabular}{|c|c|c|c|c|c|c|}
\hline Métodos gerenciais & $\begin{array}{c}\text { Não há } \\
\text { planos } \\
\text { para } \\
\text { adotar } \\
(\%)\end{array}$ & $\begin{array}{c}\text { Avaliou e } \\
\text { descartou a } \\
\text { adoção (\%) }\end{array}$ & $\begin{array}{c}\text { Em } \\
\text { processo } \\
\text { de } \\
\text { avaliação } \\
\text { (\%) }\end{array}$ & $\begin{array}{c}\text { Adoção } \\
\text { inicial } \\
(\%)\end{array}$ & $\begin{array}{c}\text { Adoção } \\
\text { plena } \\
(\%)\end{array}$ & Mediana \\
\hline Custeio Padrão & 28 & 6 & 19 & 22 & 25 & 3 \\
\hline Custeio por Absorção & 3 & 0 & 6 & 38 & 53 & 5 \\
\hline Custeio Variável & 38 & 6 & 9 & 28 & 19 & 3 \\
\hline Descentralização & 34 & 3 & 9 & 13 & 41 & 4 \\
\hline Moeda Constante & 28 & 3 & 3 & 9 & 56 & 5 \\
\hline Orçamento & 3 & 0 & 6 & 25 & 66 & 5 \\
\hline Preço de Transferência & 34 & 13 & 16 & 13 & 25 & 3 \\
\hline Retorno sobre Investimento & 44 & 16 & 22 & 13 & 6 & 2 \\
\hline Valor Presente & 44 & 19 & 19 & 9 & 9 & 2 \\
\hline BSC & 44 & 6 & 0 & 13 & 34 & 3 \\
\hline Benchmarking & 44 & 0 & 3 & 3 & 50 & 5 \\
\hline$A B C$ & 41 & 3 & 6 & 9 & 41 & 4 \\
\hline Custeio Meta & 41 & 3 & 13 & 22 & 22 & 3 \\
\hline$A B M$ & 44 & 9 & 6 & 28 & 13 & 2 \\
\hline EVA & 41 & 28 & 13 & 16 & 3 & 2 \\
\hline VBM & 47 & 41 & 0 & 13 & 0 & 2 \\
\hline JIT & 56 & 34 & 9 & 0 & 0 & 1 \\
\hline Kaizen & 50 & 47 & 3 & 0 & 0 & 2 \\
\hline Planejamento Estratégico & 28 & 0 & 6 & 16 & 50 & 5 \\
\hline Simulação & 38 & 0 & 3 & 13 & 47 & 4 \\
\hline Teoria das Restrições & 53 & 41 & 3 & 3 & 0 & 1 \\
\hline
\end{tabular}

Obs.: Med = Mediana das respostas obtidas na escala de 1 a 5

Fonte: Dados da pesquisa. 
Dentre os principais resultados evidenciados pela Tabela 5, pode-se observar que os principais métodos utilizados pelos responsáveis pela gestão dos hotéis em sua forma plena compreendem ao Orçamento empresarial com $66 \%$, a utilização da Moeda Constante com 56\%, o método de Custeio por Absorção com 53\%, a análise do Benchmarking com 50\%, o estabelecimento do Planejamento Estratégico com 50 \%, a análise da Simulação de resultados com 47\%, a estrutura com forma de Descentralização com 41\%, e o método de Custeio Baseado em Atividades $[A B C]$. Os resultados revelam uma adoção maior de métodos tradicionais, o que corrobora com os achados de Faria et al. (2012), sendo que neste estudo conduzido na região do Algarve, Portugal, predominou a utilização do Orçamento.

Em contra partida, aparecem os métodos gerenciais que os gestores não têm planos de adoção futura, que compreendem principalmente o JIT [Just in time] com 56\%, a Teoria das Restrições com 53\%, o método Kaizen com 50\%, a Gestão Baseada em Valor [VBM] com 47\%, o Retorno sobre Investimento [ROI] com 44\%, a técnica de análise do Valor Presente com 44\%, a Gestão Baseada em Atividade [ABM] com 44\% e o método de análise do EVA [Economic Value added] com $41 \%$.

Os métodos que ficaram com uma mediana 3, entre a adoção plena, parcial, ou sem planos de adoção correspondeu ao Balanced Scorecard [BSC] com 44\% dos respondentes da pesquisa sem planos de adoção e outros 34\% já com adoção plena deste artefato. O Custeio Meta com 41\% dos hotéis sem planos de adoção, o Custeio Variável com 38\% dos hotéis sem planos de adoção, o Preço de Transferência com 34\% da amostra investigada sem planos de adoção e o Custeio Padrão com 28\% dos hotéis sem planos de adoção e outros $25 \%$ com adoção completa deste método de custeio. No estudo de Faria et al. (2012) o BSC apresentou baixa utilização, como também outros métodos considerados modernos.

A frente destes resultados, foram pesquisados os fatores comportamentais da TIPI destacados na Tabela 6, presentes nos indivíduos responsáveis pelo processo de gestão dos hotéis da amostra da pesquisa, para posterior análise da relação com os métodos gerenciais. 
Tabela 6 - Fatores comportamentais presentes no processo de gestão dos hotéis pesquisados.

\begin{tabular}{|c|c|c|c|c|c|c|c|}
\hline Fator comportamental & Sigla & $\begin{array}{c}\text { Muito } \\
\text { pouco ou } \\
\text { quase nada } \\
\text { (\%) }\end{array}$ & $\begin{array}{l}\text { Pouco } \\
\text { ou nada } \\
(\%)\end{array}$ & Inseguro (\%) & $\begin{array}{l}\text { Alto } \\
\text { ou } \\
\text { Total } \\
(\%)\end{array}$ & $\begin{array}{l}\text { Muito } \\
\text { alto ou } \\
\text { total } \\
\text { (\%) }\end{array}$ & Med. \\
\hline Aberto a novas experiências. & \multirow{2}{*}{$A$} & 3 & 31 & 6 & 22 & 38 & 4 \\
\hline Convencional, sem criatividade. & & 3 & 38 & 13 & 38 & 9 & 3 \\
\hline Autodisciplinado. & \multirow{2}{*}{$C$} & 13 & 28 & 0 & 19 & 41 & 4 \\
\hline Desorganizado, descuidado. & & 44 & 25 & 3 & 25 & 3 & 2 \\
\hline Extrovertido, entusiasta. & \multirow{2}{*}{$\mathrm{E}$} & 0 & 34 & 3 & 19 & 44 & 4 \\
\hline Reservado, quieto. & & 53 & 19 & 9 & 19 & 0 & 1 \\
\hline Simpático. & \multirow{2}{*}{ S } & 3 & 22 & 9 & 47 & 19 & 4 \\
\hline Crítico. & & 25 & 6 & 13 & 41 & 16 & 4 \\
\hline Calmo, emocionalmente estável. & & 6 & 28 & 0 & 50 & 16 & 4 \\
\hline Ansioso, chateia-se facilmente. & & 47 & 13 & 0 & 31 & 9 & 2 \\
\hline \multicolumn{8}{|c|}{$\begin{array}{c}\text { Legenda: } \mathrm{A}=\text { Abertura; } \mathrm{C}=\text { Consciência; } \mathrm{E}=\text { Extroversão; } \mathrm{S}=\text { Sociabilidade ou Agradabilidade; } \mathrm{N}= \\
\text { Neuroticismo } \\
\text { Obs.: } \mathrm{Med}=\text { Mediana das respostas obtidas na escala de } 1 \text { a } 5\end{array}$} \\
\hline
\end{tabular}

Fonte: Dados da pesquisa.

Conforme a Tabela 6, destaca-se que em relação ao grau de Abertura dos gestores dos hotéis pesquisados que 38\% apresenta-se com características individuais aberta para novas experiências, resultado que indica que os gestores a luz dessa característica estão mais propensos a utilizarem-se de ferramentas modernas no processo de gestão das organizações hoteleiras, ou seja, apresentam-se com maior grau explorador $(A+)$.

Entretanto, ainda quanto a Abertura observou-se uma proximidade nos resultados que indicam que $31 \%$ dos indivíduos pesquisados apresentaramse com pouco ou nada de abertura para novas experiências, resultado que demonstra que na amostra pesquisada os gestores dos hotéis ainda estão no meio termo agindo de forma convencional (38\% pouco ou nada; $38 \%$ alto ou total) no que se refere-se a utilizar-se da criatividade para uso na gestão ou se trabalham com uma visão tradicional na gestão do hotel, sem arriscar no gerenciamento da organização. 
No que se remete ao fator comportamental da Consciência, pode-se observar que os gestores investigados apresentam-se com características mais flexíveis (C-), em razão de que apresentaram-se mais organizados (44\% muito pouco ou quase nada desorganizado) e disciplinados (41\% muito alto ou totalmente autodisciplinados).

Em relação ao grau de Extroversão dos gestores dos hotéis pesquisados, observa-se que 44\% apresenta-se com características individuais voltadas para o aspecto extrovertido e entusiasta, porém seguido de indivíduos com pouco ou nada de extroversão (34\%). De modo que a maioria dos gestores pesquisados (54\%) apontou ser muito pouco ou quase nada reservado, resultado que implica em fortes características voltadas a Extroversão $(E+)$.

No que tange a Sociabilidade ou Agradabilidade, destaca-se que a amostra da pesquisa apresentou-se $47 \%$ com características voltadas a simpatia, que de acordo com a literatura indica o grau de parceria dos gestores hoteleiros, fato que corrobora que os mesmos estão mais propensos a trabalhar com parcerias na condução da gestão dos hotéis, ou seja, apresentam-se com maior grau de parceria $(\mathrm{S}+)$.

Entretanto, ainda quanto a Sociabilidade ou Agradabilidade observou-se que $41 \%$ dos indivíduos pesquisados apresentaram-se com alto ou total grau de criticidade, o que implica em um alto nível de competição presente nos gestores hoteleiros.

QuantoaoNeuroticismo, pode-seinferirqueaamostrapesquisadaapresentouse mais reativa $(\mathrm{N}+), 50 \%$ Alto ou totalmente calmo ou emocionalmente estável, do que resiliente, $47 \%$ muito pouco ou quase nada ansioso. Resultado implica que os respondentes possuem características de serem pessoas mais calmas e seguras, sendo que não praticam ações em função de sentimentos e emoções, mas direcionam suas ações em detrimento de circunstancias que sejam concretas e reais.

Os resultados acima revelam que os respondentes estão abertos para novas experiências, com características mais flexíveis, apresentam-se mais organizados e disciplinados, são pessoas que tem preferência por estar perto de outras, e ainda, é comum participarem de diversas atividades. Estes resultados 
corroboram com os argumentos de Rouwelaar e Bots (2008) que mostram que as características abertura, sociabilidade e extroversão apresentam uma associação positiva com o grau de envolvimento do gestor, como também coaduna com os achados de Teles et al (2012) de que os pesquisados revelaram afinidade para atividades desenvolvidas em grupo, centradas em habilidades para tomada de decisão, competência de solucionar problemas e de liderança.

Diante desses achados, a Tabela 7 apresenta o resultado obtido com o cálculo da correlação canônica entre o conjunto de variáveis do grupo dos fatores comportamentais [TIPI] com o conjunto de variáveis do grupo dos métodos gerenciais utilizados no processo de gestão das organizações empresariais.

Tabela 7 - Correlação canônica dos Fatores Comportamentais

\section{Fator Comportamental de Abertura (A)}

\begin{tabular}{c|c|c|c|c|c|c}
\hline Número & Autovalor & $\begin{array}{c}\text { Canonical } \\
\text { Correlação }\end{array}$ & $\begin{array}{c}\text { Wilks } \\
\text { Lambda }\end{array}$ & Chi-quadrado & D.F & P-Value \\
\hline 1 & 0,9590 & 0,9793 & 0,0086 & 90,4536 & 42 & $\mathbf{0 , 0 0 0 0}$ \\
\hline 2 & 0,7912 & 0,8895 & 0,2088 & 29,763 & 20 & 0,0738 \\
\hline
\end{tabular}

Fator Comportamental de Consciência (C)

\begin{tabular}{c|c|c|c|c|c|c}
\hline Número & Autovalor & $\begin{array}{c}\text { Canonical } \\
\text { Correlação }\end{array}$ & $\begin{array}{c}\text { Wilks } \\
\text { Lambda }\end{array}$ & Chi-quadrado & D.F & P-Value \\
\hline 1 & 0,9806 & 0,9903 & 0,0094 & 88,606 & 42 & $\mathbf{0 , 0 0 0 0}$ \\
\hline 2 & 0,5136 & 0,7166 & 0,4864 & 13,6922 & 20 & 0,8458 \\
\hline
\end{tabular}

Fator Comportamental de Extroversão (E)

\begin{tabular}{c|c|c|c|c|c|c}
\hline Número & Autovalor & $\begin{array}{c}\text { Canonical } \\
\text { Correlação }\end{array}$ & $\begin{array}{c}\text { Wilks } \\
\text { Lambda }\end{array}$ & Chi-quadrado & D.F & P-Value \\
\hline 1 & 0,9136 & 0,9558 & 0,0572 & 54,3486 & 42 & 0,0959 \\
\hline 2 & 0,3375 & 0,5810 & 0,6625 & 7,8234 & 20 & 0,9930 \\
\hline
\end{tabular}

Fator Comportamental de Sociabilidade ou Agradabilidade (S)

\begin{tabular}{c|c|c|c|c|c|c}
\hline Número & Autovalor & $\begin{array}{c}\text { Canonical } \\
\text { Correlação }\end{array}$ & $\begin{array}{c}\text { Wilks } \\
\text { Lambda }\end{array}$ & Chi-quadrado & D.F & P-Value \\
\hline 1 & 0,8800 & 0,9381 & 0,0418 & 60,3326 & 42 & $\mathbf{0 , 0 3 3 1}$ \\
\hline 2 & 0,6519 & 0,8074 & 0,3481 & 20,0514 & 20 & 0,4547 \\
\hline \multicolumn{7}{c}{ Fator Comportamental de Neuroticismo (N) } \\
\hline Número & Autovalor & $\begin{array}{c}\text { Canonical } \\
\text { Correlação }\end{array}$ & $\begin{array}{c}\text { Wilks } \\
\text { Lambda }\end{array}$ & Chi-quadrado & D.F & P-Value \\
\hline 1 & 0,9872 & 0,9936 & 0,0064 & 95,8494 & 42 & $\mathbf{0 , 0 0 0 0}$ \\
\hline 2 & 0,4956 & 0,7040 & 0,5044 & 13,0018 & 20 & 0,8773 \\
\hline
\end{tabular}

Fonte: Dados da pesquisa.

Com base na Tabela 7, observa-se que o grupo das variáveis que representam os fatores comportamentais da TIPI, dada a análise da correlação canônica combinada aos métodos gerenciais utilizados no processo de gestão dos hotéis, a partir da primeira combinação linear calculada pelo modelo obtiveram 
coeficientes de correlação considerados de acordo com Hair Jr, Black, Babin, Anderson, \& Tatham (2009) com uma força de associação muito forte, variando entre 0,91 a 1,00 .

Entretanto, destaca-se que as combinações lineares significativas foram observadas para os respectivos fatores comportamentais: Abertura com significância ao nível de 1\%, Consciência ao nível de 1\%, Sociabilidade ou Agradabilidade ao nível de $5 \%$ e para o fator do Neuroticismo ao nível de $1 \%$. Sendo que não foram obtidas combinações lineares significativas estatisticamente para o fator comportamental referente a Extroversão, conforme pode-se observar nos valores representados pelo $P$-Value.

Assim, para a observação dos coeficientes de correlação para as variáveis canônicas, neste estudo são utilizados a primeira combinação linear de cada grupo analisado, baseada na maior correlação evidenciada entre as combinações calculadas pelo modelo. Dessa forma, a Tabela 8 apresenta os respectivos resultados referentes aos coeficientes apurados.

Tabela 8 - Coeficientes para as variáveis canônicas do grupo dos Fatores Comportamentais e do grupo dos Métodos Gerenciais

\begin{tabular}{|c|c|c|c|c|c|c|}
\hline Grupos & Variáveis & \multicolumn{5}{|c|}{ Coeficientes combinação Linear Significativas } \\
\hline & & \multicolumn{5}{|c|}{ Fatores comportamentais } \\
\hline Grupo dos Métodos Gerenciais & Questão & $A$ & C & $E^{*}$ & $S$ & $N$ \\
\hline Custeio Padrão & Q23 & 0,3661 & 0,2450 & 0,4804 & 0.2262 & 0.3028 \\
\hline Custeio por Absorção & Q24 & 0,6498 & 0,1963 & 0,4630 & 0.6328 & -0.0079 \\
\hline Custeio Variável & Q25 & $-0,4684$ & 0,0315 & 0,3859 & 0.2651 & -0.0054 \\
\hline Descentralização & Q26 & 0,0794 & 0,1540 & 0,0483 & -0.0127 & 0.2170 \\
\hline Moeda Constante & Q27 & $-0,1202$ & 0,0246 & $-0,0448$ & -0.3577 & -0.0199 \\
\hline Orçamento & Q28 & 0,1489 & 0,1500 & $-0,0343$ & 0.1381 & -0.0471 \\
\hline Preço de Transferência & Q29 & 0,1362 & $-0,0696$ & $-0,0639$ & 0.4364 & 0.1861 \\
\hline Retorno sobre Investimento & Q30 & $-0,0764$ & 0,0346 & $-0,0608$ & -0.4385 & 0.1299 \\
\hline Valor Presente & Q31 & 0,2329 & 0,2345 & 0,2967 & 0.0236 & -0.0582 \\
\hline Balanced Scorecard & Q32 & $-1,7039$ & $-0,6076$ & $-1,1930$ & $-2,7765$ & -0.5455 \\
\hline Benchmarking & Q33 & 0,1303 & $-0,4691$ & $-0,0613$ & 1,9917 & 0.1105 \\
\hline$A B C$ & Q34 & 0,9245 & 0,4086 & $-0,0751$ & -0.4271 & -0.0277 \\
\hline Custeio Meta & Q35 & 0,2045 & 0,0731 & 0,0754 & 0.4077 & 0.1137 \\
\hline Gestão Baseada em Atividade & Q36 & 0,3313 & 0,2384 & 0,9641 & $-1,0214$ & 0.4024 \\
\hline
\end{tabular}


ISSN: 1983-7151

\begin{tabular}{|c|c|c|c|c|c|c|}
\hline EVA (Economic Valueadded) & Q37 & $-0,1034$ & $-0,1491$ & $-0,2507$ & -0.4644 & -0.2588 \\
\hline Gestão Baseada em Valor & Q38 & $-0,0221$ & $-0,0486$ & $-0,2578$ & -0.1346 & 0.0664 \\
\hline JIT (Just in time) & Q39 & $-0,0347$ & $-0,0058$ & 0,2224 & 0.3702 & -0.0716 \\
\hline Kaizen & Q40 & 0,2970 & 0,2252 & 0,0007 & -0.6838 & -0.0520 \\
\hline Planejamento Estratégico & Q41 & $-0,2411$ & $-0,0222$ & 0,0253 & -0.3649 & -0.1069 \\
\hline Simulação (de resultados) & Q42 & 0,8213 & 0,7253 & 0,3602 & 0.7614 & 0.8115 \\
\hline Teoria das Restrições & Q43 & $-0,2335$ & $-0,2122$ & 0,0352 & 0.1647 & -0.2181 \\
\hline Fatores comportamentais & Questão & & & & & \\
\hline Abertura $(\mathrm{A}+)$ & Q13 & 0,9984 & - & - & - & - \\
\hline Abertura (A-) & Q14 & $-0,0035$ & - & - & - & - \\
\hline Consciência $(C+)$ & Q15 & - & 0,8757 & - & - & - \\
\hline Consciência (C-) & Q16 & - & $-0,1453$ & - & - & - \\
\hline Extroversão $(E+)$ & Q17 & - & - & 0,9657 & - & - \\
\hline Extroversão (E-) & Q18 & - & - & $-0,0417$ & - & - \\
\hline $\begin{array}{c}\text { Sociabilidade/Agradabilidade } \\
\qquad(\mathrm{S}+)\end{array}$ & Q19 & - & - & - & $-0,4177$ & - \\
\hline $\begin{array}{c}\text { Sociabilidade/Agradabilidade } \\
\text { (S-) }\end{array}$ & Q20 & - & - & - & 1,3251 & - \\
\hline Neuroticismo $(\mathrm{N}+)$ & Q21 & - & - & - & - & 0,1357 \\
\hline Neuroticismo (N-) & Q22 & - & - & - & - & $-0,9388$ \\
\hline \multicolumn{2}{|c|}{ Correlação Canônica } & 0,9793 & 0,9903 & 0,9558 & 0,9381 & 0,9936 \\
\hline
\end{tabular}

Fonte: Dados da pesquisa.

De acordo com a Tabela 8, pode-se destacar que em relação ao fator comportamental de Abertura que o menor grau de adoção do Balanced Scorecard [BSC] é determinada pelo grau em que o responsável pela gestão do hotel apresenta-se com características comportamentais conservadoras. De modo, que o maior grau de utilização do Custeio Baseado em Atividades $[A B C]$ está determinado pela característica mais exploradora dos gestores dos hotéis. Assim, esse resultado implica que a partir da amostra pesquisada, a gestão realizada por indivíduos com características comportamentais mais conservadoras, apresentam respectivamente maior resistência à utilização de métodos gerenciais modernos. Enquanto que os indivíduos que são mais exploradores, com maior nível de Abertura, tendem a aplicar técnicas modernas para auxiliar no processo de gestão dos hotéis.

No que tange ao fator comportamental de Consciência, infere-se que na amostra pesquisada identificou-se que menor grau de utilização do Balanced Scorecard [BSC], está associando ainda a menor flexibilidade dos responsáveis pela gestão. Sendo que o maior grau de utilização de simulação de resultados, 
provisão de resultados, está determinado pela característica comportamental do indivíduo com maior grau de disciplinariedade.

No fator da Extroversão, observa-se resultados inconclusivos, mas que sinalizam a que o menor grau de utilização do Balanced Scorecard [BSC] está ligado ao maior grau que o indivíduo responsável pela gestão é introvertido. Ainda, destaca-se que o grau de utilização da Gestão Baseada em Atividade [ABM] é determinada de acordo com o fator comportamental do indivíduo mais extrovertido. Assim, esse resultado implica que o grupo de respondentes que detém preferencias por atuarem sozinhas, que são calmas, sérias e ainda reservadas, possuem uma baixa utilização do BSC, já os que apresentam comportamentos que demonstrem a necessidade de estar perto de outras e mostram capacidade de participação em diversas atividades, fazem um uso maior da ABM.

Observando o Fator Comportamental de Sociabilidade ou Agradabilidade o menor grau de utilização do Balanced Scorecard [BSC] é ainda determinado pela característica do indivíduo que apresenta-se com menor competitividade. De modo que, a maior utilidade em relação a observação do Benchmarking do mercado é determinado com base na amostra pesquisada pelos indivíduos que apresentam personalidade voltada ao grau de parceria que busca estabelecer no mercado.

Por fim, em relação ao Fator Comportamental de Neuroticismo, evidenciouse que o menor grau de utilização do Balanced Scorecard [BSC] está determinado pelo menor grau que o indivíduo é resiliente. E que a maior utilização da simulação de resultados está determinada pelo grau de reatividade do indivíduo responsável pela gestão.

Diante destes resultados, pode-se observar que o menor grau de utilização do Balanced Scorecard [BSC] está determinado pelos fatores comportamentais da TIPI, resultado que implica que respondentes que detém comportamento calmo, seguro e que apresentam ações em detrimento de fatos concretos e reais, são os menos propícios a fazerem uso do BSC e os que são mais inseguros, preocupados, atentos e emotivos, apresentam mais estresse e capacidade reativa, dessa forma, tendem a fazer um uso maior do artefato simulação de resultado. 


\section{CONSIDERAÇÕES FINAIS}

O presente estudo teve como objetivo verificar a relação dos fatores comportamentais na utilização dos métodos de contabilidade gerencial nos hotéis de Santa Catarina. Assim, os resultados da pesquisa destacam que os fatores comportamentais são altamente correlacionados as escolhas dos gestores que levam ao grau de adoção dos métodos nas organizações hoteleiras pesquisadas do Estado de Santa Catarina.

Os achados destacam que os gestores dos hotéis investigados apresentamse principalmente com fatores comportamentais voltados a uma visão com maior grau explorador $(A+)$ em relação ao nível de Abertura do gestor, maior grau de flexibilidade (C-) em relação a Consciência, maior grau de Extroversão $(\mathrm{E}+)$ em se tratando da Extroversão, maior grau de parceria $(\mathrm{S}+)$ no que tange a Sociabilidade e Agradabilidade e maior grau de reatividade $(\mathrm{N}+)$ em relação ao Neuroticismo.

Sob o qual, estes fatores comportamentais levaram a identificar que o grau de utilização dos métodos gerenciais modernos, principalmente o menor uso do instrumento do Balanced Scorecard [BSC] é determinado pelos gestores que apresentam-se com suas características individuais mais conservadoras, com menor flexibilidade, maior grau de introversão, com menor grau competitividade e pelo menor grau de resiliência do responsável pela gestão do hotel.

Dessa forma, conclui-se que os fatores comportamentais influenciam na utilização dos métodos de contabilidade gerencial nas organizações hoteleiras, de modo que pode-se observar uma alta correlação entre as variáveis analisadas, corroborando com os estudos desenvolvidos por Gosling et al. (2003). Ampliando ainda os achados dos estudos realizados no contexto dos hotéis por Teles et al. (2012) que analisaram os fatores comportamentais dos gestores e de Faria et al. (2012) que exploraram as práticas de custeio e controle de gestão. Assim, a presente pesquisa contribui para o entendimento mais detalhado da relação entre os fatores comportamentais e a utilização dos métodos gerenciais em hotéis, bem como os achados desta pesquisa contribuem para o desenvolvimento de informações para novas pesquisas. 
Diante desse aspecto, como limitações da pesquisa destaca-se o número de retorno dos questionários encaminhados a população da pesquisa. Como sugestão para a realização de trabalhos futuros, recomenda-se o uso de outras técnicas de análise estatística dos dados, bem como aplicação deste estudo em outras regiões do país para obter-se mais evidencias quanto aos fatores comportamentais e sua relação na utilização dos métodos gerenciais na gestão do setor hoteleiro.

\section{REFERÊNCIAS}

Associação Brasileira da Indústria de Hotéis do Estado de Santa Catarina. Hotéis associados (n.d.) Hotéis Associados. Recuperado em 02 outubro, 2015, de http://www.abih-sc.com.br/ hoteis-associados.

Adler, R., Everett, A. M., \& Waldron, M. (2000). Advanced management accounting techniques in manufacturing: utilization, benefits, and barriers to implementation. In Accounting Forum, Blackwell Publishers Ltd., 24 (2), 131-150 .

Almeida, M. C. (1996) Auditoria: um curso moderno e completo (5a ed.) São Paulo: Atlas.

Araujo, A. O. (2001) Contribuição ao estudo de indicadores de desempenho de empreendimentos hoteleiros, sob o enfoque da gestão estratégica. Tese de doutorado, Universidade de São Paulo, São Paulo, SP, Brasil.

Bandura, A. (1977) Social learning theory. Englewood Cliffs, N. J.: Prentice-Hall.

Beuren, I. M., \& Fiorentin, M. (2014). Influência de Fatores Contingenciais nos Atributos do Sistema de Contabilidade Gerencial: um estudo em empresas têxteis do Estado do Rio Grande do Sul. Revista de Ciências da Administração, 16 (38), 195-212.

Borinelli, M. L. (2006) Estrutura conceitual básica de controladoria: sistematização à luz da teoria e da práxis. Tese de doutorado, Universidade de São Paulo, São Paulo, Brasil.

Bower, J. B. (1957) A profession of accounting-or of accountancy? The Accounting Review, 32, 194-199.

Cabrelli, F. L., Ferreira, A., \& Contábeis, C. (2007). Contabilidade gerencial como ferramenta no processo de tomada de decisão. Revista Cientifica Eletrônica de Ciências Contábeis, 5 (09), 1-7.

Castro, J. K.; Santos, G. R.; Souza, P.; Lunkes, R. J. (2012) Práticas orçamentárias aplicadas em empresas hoteleiras no Brasil: um estudo na cidade de Florianópolis. Revista Estudios y Perspectivas em Turismo. 21 (4), 279-294. 
Codesso, M. M., Lunkes, R. J., \& Suave, R. (2013). Práticas orçamentárias aplicadas em empresas hoteleiras no Brasil: um estudo na cidade de Balneário Camboriú-SC. TurismoVisão e Ação, 15(2), 279-294.

Cunha, D. R., \& Frezatti, F. (2002). Gestão baseada em valor: uma pesquisa no setor hoteleiro do Rio Grande do Norte. Revista Eletrônica de Administração, 10(4), 1-18.

Digman, J. M. (2002). Personality structure: Emergence of the five-factor model. Annual review of psychology, 41(1), 417-440.

Emsley, D., \& Chung, L. H. (2010). How Management Accountants' Cognitive Style and Role Involvement Combine to Affect the Effort Devoted to Initiating Change. Abacus, 46(3), 232-257.

Faria, A. R., Trigueiros, D., \& Leonor, F. (2012). Práticas de custeio e controlo de gestão no sector hoteleiro do Algarve. Tourism \& Management Studies, 1(8), 100-107.

Ferreira, A. \& Otley, D. (2006) Exploring inter and intra-relationships between the design and use of management control systems. SSRN.

Fitzsimmons, J. A. \& Fitzsimmons, M. J. (2014) Administração de serviços: operações, estratégia e tecnologia da informação (7a ed.) Editora McGraw Hill: Porto Alegre.

Francisco Carvalho de, L., Nunes, M. F. O., Primi, R., \& Silva Nunes da, C. H. S. (2012). Evidências desfavoráveis para avaliação da personalidade com um instrumento de 10 itens. Paidéia, 22(51), 63-71.

Frezatti, F. (2005) Management accounting profile of firms located in Brazil: a field study. Brazilian Administration Review, 2 (1), 73-87.

Frezatti, F., Aguiar, A. B. D., \& Guerreiro, R. (2007). Differences between financial and management accounting: an empirical research based on the work of researchers from several countries. Revista Contabilidade \& Finanças, 18(44), 9-22.

Fonseca, J. S. \& Martins, G. A. (1996) Curso de estatística - GED. São Paulo: Atlas.

Guerreiro, R., Frezatti, F., Lopes, A. B., \& Pereira, C. A. (2005). O entendimento da contabilidade gerencial sob a ótica da teoria institucional. Organizações \& Sociedade, 12(35), 91-106.

Guerreiro, R., Cornachione Júnior, E. B., \& Soutes, D. O. (2011). Empresas que se destacam pela qualidade das informações a seus usuários externos também se destacam pela utilização de artefatos modernos de contabilidade gerencial?. Revista Contabilidade \& Finanças, 22(55), 88-113.

Gosling, S. D., Rentfrow, P. J., \& Swann, W. B. (2003). A very brief measure of the Big-Five personality domains. Journal of Research in personality, 37(6), 504-528. 
Hall, C. S., Lindzey, G., \& Campbell, J. B. (2000) Teorias da personalidade (4a ed.) Porto Alegre: Artmed.

Harden, G. (1995) The development of standards of competence in accounting. Accounting Education, 4 (1), p. 17-28.

Hair Jr, J. F., Black, W.C., Babin, B.J., Anderson, R.E., \& Tatham, R.L. (2009) Análise multivariada de dados (6a ed.) Porto Alegre: Bookman.

Horngren, C. T., Foster, G., \& Datar, S. (2004) Contabilidade de custos (11a ed.) São Paulo: Prentice Hall.

Howard, P. J. \& Howard, J. M. (1995) The big five quickstart: An introduction to the FiveFactor Model of Personality for Human Resource professionals. Centre for Applied Cognitive Studies, Charlotte.

Iudícibus, S. (1998) Contabilidade Gerencial (6a ed.) São Paulo: Atlas.

Jiambalvo, J. (2002) Contabilidade Gerencial (Quírico, T. C., Trad.) São Paulo: LTC.

Lucas, R. E., Diener, E., Grob, A., Suh, E. M., \& Shao, L. (2000) Cross-cultural evidence for the fundamental features of extraversion. Journal of Personality and Social Psychology, 79 (3), 452-468.

Lunkes, R. J. (2009) O uso de controles internos: um estudo em empresas hoteleiras. Revista de Contabilidade da UFBA, 2 (3), 50-66.

Marquesan, F. F. S., Guzzo, R. F., Zawislak, P. A., \& Tello-Gamarra, J. (2015) A importância dos ativos específicos na diferenciação de firmas do setor hoteleiro. E\&G Economia e Gestão, 15 (41), 79-111.

Martin, N. C. (2002) Da contabilidade à Controladoria: a evolução necessária. Revista Contabilidade \& Finanças, 13 (28), 7-28.

Mayer, J. D. (2005) A tale of two visions: Can a new view of personality help integrate psychology? American Psychologist, 60 (4), 294-307.

Mia, L. \& Patiar, A. (2001) The use of management accounting systems in hotels: an exploratory study. International Journal of Hospitality Management, 20 (2), 111-128.

Mondo, T. S \& Costa, J. I. P. (2013) A Influência da promoção de vendas na captação de clientes: um estudo na hotelaria catarinense. REMark-Revista Brasileira de Marketing, 12 (2), 87-107.

Mosimann, C. P. \& Fisch, S. (1999) Controladoria (2a ed.) São Paulo: Atlas.

Nascimento, A. M. \& Reginato, L. (2010) Controladoria: Instrumento de apoio ao processo decisório. São Paulo: Atlas. 
Oliveira, J., Pereira, S., \& Ribeiro, J. (2008) Investigação em contabilidade de gestão. In M. J. Major \& R. Vieira (Orgs.). Contabilidade e Controlo de Gestão: Teoria, Metodologia e Prática. Escolar Editora: Lisboa.

Pavlatos, O. \& Paggios, I. (2009) A survey of factors influencing the cost system design in hotels. International Journal of Hospitality Management, 28 (2), 263-271.

Pellinen, J. (2003) Making price decisions in tourism enterprises. International Journal of Hospitality Management, 22 (1), 217-235.

Petrocchi, M. (2007) Hotelaria: planejamento e gestão (2a ed.) São Paulo: Pearson Prentice Hall.

Rosa, F. S. \& Lunkes, R. J. (2006) Diagnóstico sobre a avaliação de desempenho em empresas hoteleiras: uma contribuição ao estudo sobre a elaboração de balanced scorecard. Anais do Simpósio de Excelência em Gestão e Tecnologia.

Robins, R. W., Tracy, J. L., Trzesniewski, K., Potter, J., \& Gosling, S. D. (2001) Personality correlates of selfesteem. Journal of Research in Personality, 35 (4), 463-482.

Rouwelaar, H. \& Bots, J. (2008) Business unit controller involvement in management: An empirical study in the Netherlands. Breukelen: Financial Controllers in Health Care.

Rutherford, D. G. (2004) Hotel: gerenciamento e operações (2a ed.) Editora Roca: São Paulo.

Sharma, D. (2002) The differential effect of environmental dimensionality, size and structure on budget system characteristics in hotel. Management Accounting Research, 13 (1), 101-130.

Soutes, D. O. (2006) Uma investigação do uso de artefatos da contabilidade gerencial por empresas brasileiras. Dissertação de mestrado, Universidade de São Paulo, São Paulo, Brasil.

Teles, J., Lunkes, R. J., Espejo, M. M. D. S. B., Nascimento, C. do (2012) Recursos cognitivos e competências na percepção de controllers que atuam em hotéis no sul do brasil. Revista Iberoamericana de Contabilidad de Gestión 10 (20), 1-14.

Vieira, W. Q. \& Souza, M. J. B. de (2005) Gestão de custos nos hotéis de lazer da região sul do Brasil. Turismo -Visão e Ação, 7 (3), 427-438.

Xavier, R. V. \& Carmo Filho, M. M. (2015) Uso dos artefatos de contabilidade gerencial: Comparativo entre os hospitais acreditados no brasil com os não acreditados da cidade de Manaus (AM). Revista Ambiente Contábil, 7 (2), 248-269.

Zonatto, V.C.S., Schuh, C., \& Zonatto, P.A.F. (2014) Contribuição dos Recursos Estratégicos nos Processos de Criação da Inovação em uma Rede de Cooperação Hoteleira. Revista Turismo em Análise, 25 (3), 700-732. 
Wilke, E. P. \& Rodrigues, L. C. (2013) Fontes de pressão institucional: reflexões sobre legitimidade na indústria hoteleira brasileira. Revista Brasileira de Pesquisa em Turismo, 7 (2), 337-357.

Wixon, R.; Keel, W.G.; Bedford (1943) Accountant's Handbook (2a. ed.) New York: The Ronald Press Company.

\section{Contribuição dos autores na construção do artigo}

Sant' Ana: Planejamento do artigo, introdução, referencial teórico, metodologia, elaboração do instrumento de pesquisa, análise dos resultados, considerações finais e revisão do artigo.

Santos: Planejamento do artigo, introdução, referencial teórico, metodologia, elaboração do instrumento de pesquisa, análise dos resultados, considerações finais e revisão do artigo.

Cunha: Planejamento do artigo, introdução, referencial teórico, metodologia, elaboração do instrumento de pesquisa, análise dos resultados, considerações finais e revisão do artigo. 